\title{
A Time-Domain Analysis of Intracardiac Electrograms for Arrhythmia Detection
}

\author{
LORENZO A. DICARLO, ROBERT D. THRONE, and JANICE M. JENKINS
}

From the Department of Electrical Engineering and Computer Science, School of Engineering, and the School of Medicine, University of Michigan, Ann Arbor, and the Cardiac

Electrophysiology Laboratory, St. Joseph Mercy Hospital of the Catherine McAuley Health

Center, Ann Arbor, Michigan

DICARLO, L.A., ET AL.: A Time-Domain Analysis of Intracardiac Electrograms for Arrhythmia Detection. The analysis of intracardiac electrogram morphology has been proposed as a complementary method for accurate discrimination between sinus rhythm (SR), supraventricular dysrhythmias, and ventricular dysrhythmias by automatic antitachycardia and cardioverter defibrillator devices. In this study, the performance of a traditional time-domain method for surface electrocardiogram interpretation-Correlation Waveform Analysis (CWA) and a newly developed technique-Bin Area Method (BAM) were used to analyze unfiltered intraatrial and intraventricular electrograms obtained from 47 patients during routine cardiac electrophysiology studies. Nineteen patients had 31 distinct, sustained, monomorphic ventricular tachycardias (VTs) induced; 13 patients had paroxysmal bundle branch block of supraventricular origin (BBB) induced; 19 patients had retrograde atrial activation during ventricular overdrive pacing. Three patients were common to two or more groups. Using a best fit electrogram alignment, both CWA and BAM distinguished VT from SR in 28/31 cases $(90 \%)$, BBB from SR in 15/15 patients $(100 \%)$, and anterograde from retrograde atrial activation in $19 / 19$ patients (100\%). We conclude that the use of time-domain techniques that are independent of amplitude and baseline fluctuations appear to be reliable for discrimination of retrograde atrial activation, paroxysmal BBB, and VT from SR using intracardiac electrograms. Reduction of computational time and power constraints, without sacrificing reliable dysrhythmia discrimination, is possible. These features may make real-time morphology analysis of intracardiac electrograms feasible for automatic antitachycardia and cardioverter-defibrillator devices. (PACE, Vol. 14, February, Part II 1991)

arrhythmia analysis, computer recognition of arrhythmia

\section{Introduction}

The use of rate and rate variation alone by currently available automatic antitachycardia and cardioverter-defibrillator devices to discriminate between sinus rhythm (SR), supraventricular dysrhythmias, and ventricular dysrhythmias has not been satisfactory. ${ }^{1-4}$ The utilization of comple-

This work was partially supported by NSF Grant No. BCS8909042.

Address for reprints: Lorenzo DiCarlo, M.D., Reichert Health Building, R-3003, Catherine McAuley Health Center, P. O. Box 994, Ann Arbor, MI 48106. mentary algorithms which analyze the morphology of intracardiac electrograms has been proposed as one means of achieving more accurate discrimination. ${ }^{6-10}$

In this study, we assessed the performance of a time-domain method traditionally utilized for surface electrocardiogram interpretation-correlation waveform analysis (CWA) and a new technique-Bin Area Method (BAM) for discrimination of retrograde atrial activation, paroxysmal bundle branch block (BBB), and ventricular tachycardia (VT) from SR with and without chronic $\mathrm{BBB}$, an intraventricular conduction delay, and/ or antiarrhythmic therapy. 


\section{Methods and Materials}

\section{Electrophysiology Study}

Bipolar $(1 \mathrm{~cm})$ atrial and ventricular endocardial electrograms were recorded during elective clinical cardiac electrophysiology studies as previously reported. ${ }^{9}$ Nineteen consecutive patients had 31 distinct, sustained, monomorphic VTs induced (group 1)(Table I); 13 patients had paroxysmal BBB of supraventricular origin induced (group 2)(Table II); 19 consecutive patients had 1:1 retrograde atrial activation during ventricular overdrive pacing (group 3)(Table III). None of the patients had dual atrioventricular nodal pathways or accessory atrioventricular connections. One patient was common to all three groups, and two patients were common to two groups.

\section{Methods of Analysis}

Recorded endocardial electrograms were subsequently replayed and digitized on a personal computer with a Tecmar Lab Master (Scientific Solutions, Inc., Solon, $\mathrm{OH}$, USA) analog-to-digital system at a sampling rate of $1000 \mathrm{~Hz}$. Programs for digitization and subsequent waveform analysis were written in the $C$ programming language and 8086 assembly language. Data sets consisted of three passages from each patient.

An initial passage of SR/AF, NSR, or anterograde atrial activation was used to construct a ventricular or atrial electrogram template by signal averaging. The template was used for subsequent comparison with a second, separate passage of SR/ $\mathrm{AF}, \mathrm{NSR}$, or anterograde control passage and a third passage of either VT (group 1), BBB (group 2), or retrograde atrial activation (group 3). A careful selection of window size effectively excluded any local repolarization in order to avoid the inclusion of injury current caused by temporary endocardial damage adjacent to the catheter. The template and the electrogram under analysis were compared using a best fit alignment.

In presenting both CWA and BAM, the following notation will be used: $N=$ the number of points in the template; $t_{i}=$ the template points; $s_{i}=$ the signal points to be processed; $\bar{t}=$ the template average; and $\bar{s}=$ the signal average.

\section{CWA}

The correlation coefficient, $\rho,{ }^{9}$ is independent of amplitude fluctuations, baseline changes, and produces an output between -1 and 1 . Mathematically, the correlation coefficient is defined as,

$$
\rho=\frac{\sum_{i=1}^{i=N}\left(t_{i}-\bar{t}\right)\left(s_{i}-\bar{s}\right)}{\sqrt{\sum_{k=1}^{k=N}\left(t_{k}-\bar{t}\right)^{2}} \sqrt{\sum_{k=1}^{k=N}\left(s_{k}-\bar{s}\right)^{2}}}
$$

\section{BAM}

BAM compares corresponding areas or bins constructed from the template with bins constructed from subsequent depolarizations using a simple error measure. Consecutive sample points are summed to estimate the areas using a rectangular area rule in equal sized bins. The average of these bin values is then removed resulting in a correction of baseline shift, and then these corrected bin values are normalized by the absolute sum of all corrected bin values. As a final step, the sum of the absolute difference of these normalized and corrected bins with an identically processed template is computed.

To form three-point bins, $S_{1}=s_{1}+s_{2}+s_{3}$, $S_{2}=s_{4}+s_{5}+s_{6} \ldots$ and $S_{M}=s_{N-2}+s_{N-1}+$ $s_{N}$.

Template points, $t_{i}$, are processed similarly to form the $T_{i}$. For $M$ equally sized bins in the template, the index of merit for BAM is given as:

$$
\rho=1-\sum_{i=1}^{i=M}\left|\frac{T_{i}-\bar{T}}{\sum_{k=1}^{k=M}\left|T_{k}-\bar{T}\right|}-\frac{S_{i}-\bar{S}}{\sum_{k=1}^{k=M}\left|S_{k}-\bar{S}\right|}\right|
$$

where

$$
\bar{S}=\frac{1}{M} \sum_{k=1}^{k=M} S_{k} \text {, and } \bar{T}=\frac{1}{M} \sum_{\substack{k=M \\ k=1}} T_{k} \text {. }
$$

Because of the design of BAM, all template processing is performed in advance, i.e., prior to comparison of the template with subsequent electrograms under analysis. BAM is designed such 
Table I

Patient Data for Discriminating VT from SR/AF

\begin{tabular}{|c|c|c|c|c|c|}
\hline Patient & Sex & $\begin{array}{c}\text { Heart } \\
\text { Disease }\end{array}$ & Drugs & $\begin{array}{c}\text { Sinus Rhythm/Atrial } \\
\text { Fibrillation QRS Morphology }\end{array}$ & $\begin{array}{c}\text { Ventricular Tachycardia } \\
\text { QRS Morphology }\end{array}$ \\
\hline 1 & $M$ & CAD & None & SR-Normal & RBB-S/R \\
\hline 2 & $M$ & CAD & None & SR-Normal & LBB-S/L \\
\hline $3 a$ & $M$ & CAD & None & SR-LBBB & LBB-S/R \\
\hline $3 b$ & & & & & LBB-S/L \\
\hline $4 a$ & $M$ & CAD & None & AF-LBBB & LBB-S/L \\
\hline $4 b$ & & & & & LBB-S/L \\
\hline $5 a$ & $M$ & CAD & Proc & SR-Normal & RBB-1/R \\
\hline $5 b$ & & & & & LBB-I/R \\
\hline $6 a$ & $M$ & CAD & Proc & SR-Normal & RBB-I/L \\
\hline $7 a$ & $F$ & CAD & $A M$ & SR-Normal & RBB-S/L \\
\hline $7 b$ & & & & & RBB-S/L \\
\hline 8 & $\mathrm{~F}$ & None & Am & SR-Normal & RBB-S/R \\
\hline 9 & $M$ & $C A D$ & Qu Me & SR-Normal & LBB-S/L \\
\hline $10 a$ & $M$ & CAD & Am & SR-LBBB & LBB-S/L \\
\hline 11 & $M$ & VHD & $\mathrm{Am}$ & SR-LBBB & LBB-S/R \\
\hline 12 & $M$ & CAD & $\mathrm{Am} \mathrm{Me}$ & SR-LBBB & LBB-I/R \\
\hline 13 & $M$ & CAD & En & SR-LBBB & LBB-I/L \\
\hline 14 & $M$ & CAD & Qu Me & SR-RBBB & RBB-S/R \\
\hline 15 & $M$ & CAD & Proc & SR-RBBB & LBB-S/R \\
\hline $16 a$ & $M$ & CAD & $A m$ & SR-IVCD & RBB-I/R \\
\hline $16 b$ & & & & & RBB-S/L \\
\hline 17 & $M$ & CAD & $A m$ & SR-IVCD & LBB-S/L \\
\hline $18 a$ & $M$ & $C A D$ & $\mathrm{Am}$ & SR-IVCD & †RBB-S/R \\
\hline $18 b$ & & & & & †LBB-S/L \\
\hline $18 c$ & & & & & †LBB-S/L \\
\hline $18 d$ & & & & & †RBB-S/R \\
\hline $18 \mathrm{e}$ & & & & & †RBB-S/R \\
\hline $10 b^{*}$ & $M$ & CAD & Am En & SR-IVCD & RBB-S/R \\
\hline $10 c$ & & & & & LBB-I/L \\
\hline $6 b^{*}$ & $M$ & CAD & En Proc & AF-IVCD & RBB-S/R \\
\hline 19 & M & CAD & Qu Di & AF-IVCD & RBB-S/L \\
\hline
\end{tabular}

* These patients were studied on two different days with two different VT morphologies. † This patient had five distinct VT morphologies. $\mathrm{CAD}=$ coronary artery disease, $\mathrm{VHD}=$ valvular heart disease $\mathrm{Am}=$ Amiodarone, $\mathrm{Di}=$ digoxin, $\mathrm{En}=$ encainide, $\mathrm{Me}=$ mexiletine, $\mathrm{Proc}=$ procainamide, $\mathrm{Qu}=$ quinidine; $\mathrm{SR}=$ sinus rhythm, $\mathrm{AF}=$ atrial fibrillation; $\mathrm{LBBB}=$ left bundle branch block, RBBB = right bundle branch block, IVCD = non-specific intraventricular conduction delay; LBB = left bundle branch morphology, $\mathrm{RBB}=$ right bundle branch morphology; $\mathrm{I}=$ inferior axis, $\mathrm{S}=$ superior axis; $\mathrm{L}=$ leftward, $\mathrm{R}=$ rightward. 
Table II.

Patient Data for Discriminating Paroxysmal Bundle Branch Block of Supraventricular Origin From Normal Sinus Rhythm

\begin{tabular}{rccllc}
\hline Patient & Sex & Heart Disease & Drugs & Method of BBB Induction & Aberration Morphology \\
\hline 20 & F & None & None & Spontaneous & RBBB \\
21 & F & None & None & AOP & RBBB \\
22 & F & None & None & AOP & RBBB \\
8 & F & None & None & Spontaneous & RBBB \\
23 & F & None & Iso & AOP & RBBB \\
24 & F & None & Iso & Atrial Fibrillation & RBBB \\
25 & F & None & Iso & Spontaneous & RBBB \\
26 & M & COPD & None & AOP & RBBB \\
27 & M & CAD & None & Spontaneous & RBBB \\
28 & M & CAD & None & AOP & RBBB \\
29 & M & CAD & Proc & AOP & LBBB \\
30 & M & None & Iso & AOP & LBBB \\
31 & M & CAD & None & AOP & \\
\hline
\end{tabular}

$\mathrm{CAD}=$ coronary artery disease, $\mathrm{COPD}=$ chronic obstructive pulmonary disease; Iso = isopril, Proc = procainamide; $\mathrm{LBBB}=$ left bundle branch block, $\mathrm{RBBB}=$ right bundle branch block; $A O P=$ atrial overdrive pacing.

\section{Table III}

Patient Data for Discriminating Retrograde from Anterograde Atrial Activation

\begin{tabular}{|c|c|c|c|c|}
\hline Patient & Sex & $\begin{array}{c}\text { Heart } \\
\text { Disease }\end{array}$ & Drugs & $\begin{array}{l}\text { Atrial Elec- } \\
\text { trode Location }\end{array}$ \\
\hline 23 & $F$ & None & Iso & RAA \\
\hline 32 & $M$ & None & None & RAA \\
\hline 33 & $M$ & None & None & RAA \\
\hline 34 & $\mathrm{~F}$ & None & None & RAA \\
\hline 35 & $M$ & CAD & None & RAA \\
\hline 36 & $M$ & None & None & RAA \\
\hline 37 & $M$ & None & None & RAA \\
\hline 38 & $\mathrm{~F}$ & None & None & RAA \\
\hline 26 & $M$ & COPD & None & RAA \\
\hline 39 & $\mathrm{~F}$ & None & None & RAA \\
\hline 40 & $M$ & CAD & None & RAA \\
\hline 41 & M & CAD & $\mathrm{Ve}$ & RAA \\
\hline 8 & $\mathrm{~F}$ & None & None & RAA \\
\hline 42 & $M$ & None & None & RAA \\
\hline 43 & $\mathrm{~F}$ & None & None & HRA \\
\hline 44 & $M$ & None & $\mathrm{Qu} \mathrm{Ve}$ & HRA \\
\hline 45 & $M$ & None & None & HRA \\
\hline 46 & $\mathrm{~F}$ & None & Ep & HRA \\
\hline 47 & $M$ & None & En & HRA \\
\hline
\end{tabular}

En = encainide, $E p=$ epinephrine, Iso $=$ isopril, $Q \mathbf{u}=$ quin idine, $\mathrm{Ve}=$ verapamil, $\mathrm{CAD}=$ coronary artery disease, $\mathrm{COPD}$ = chronic obstructive pulmonary disease, RAA = right atrial appendage, HRA = high right atrium. that it requires only one-sixth the computation of CWA for three-point bins. BAM is similar to another time-domain method of analysis-area of difference (AD). ${ }^{7}$ However, like CWA, BAM differs from $\mathrm{AD}$ in that it is independent of amplitude and baseline fluctuations.

\section{Results}

For either method, BAM or CWA, there was no universal threshold which separated VT from SR/AF, BBB from NSR, or retrograde atrial activation from anterograde atrial activation, in the patient population studied. Instead, patient specific thresholds were required for each method. Figure 1 summarizes the results of using CWA and BAM to distinguish VT from SR/AF. CWA and BAM both discriminated VT from SR/AF in 28/31 $(90 \%)$ cases. Figure 2 summarizes the results of using CWA and BAM to distinguish BBB from NSR. CWA and BAM both discriminated BBB from NSR in 13/13 (100\%) patients. Figure 3 summarizes the results of using CWA and BAM to distinguish retrograde atrial activation from anterograde atrial activation. CWA and BAM both discriminated retrograde from anterograde atrial activation in $19 / 19(100 \%)$ patients. 
PATIENT

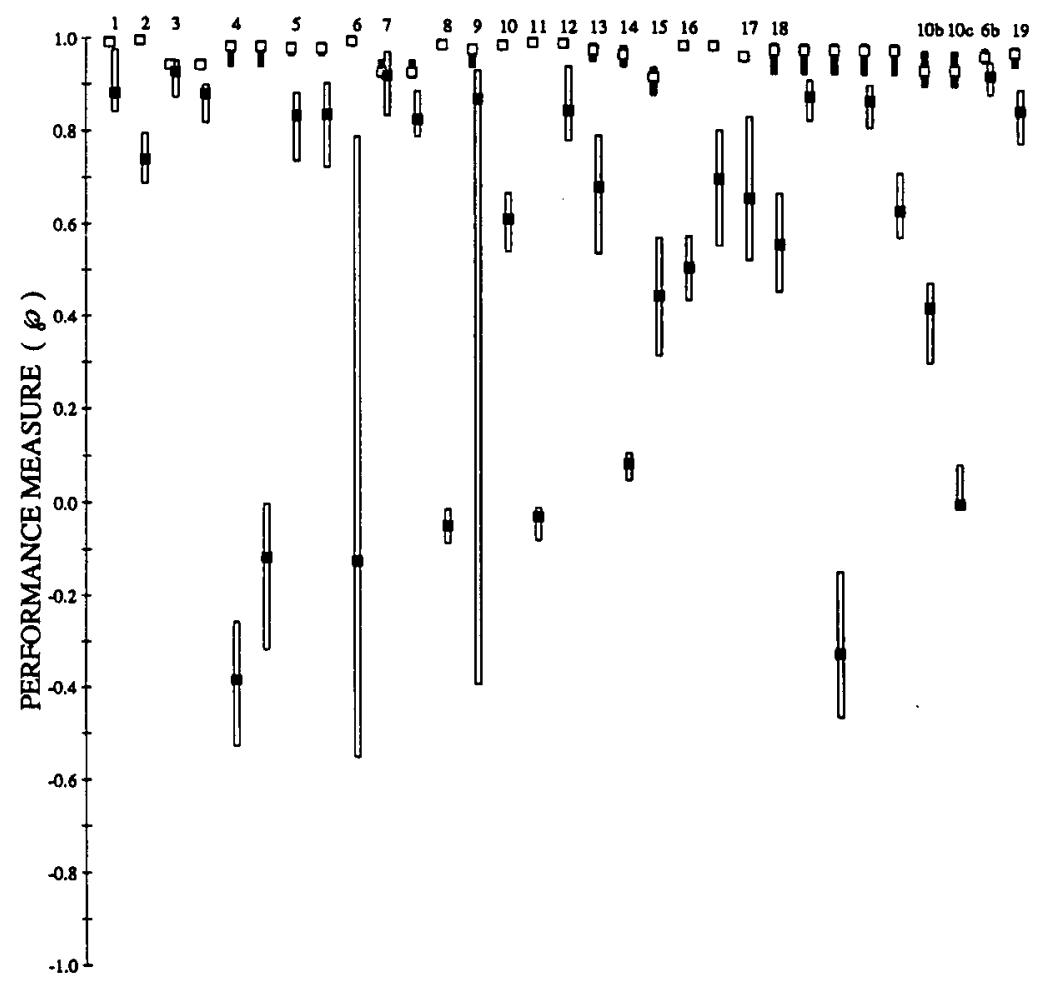

PATIENT

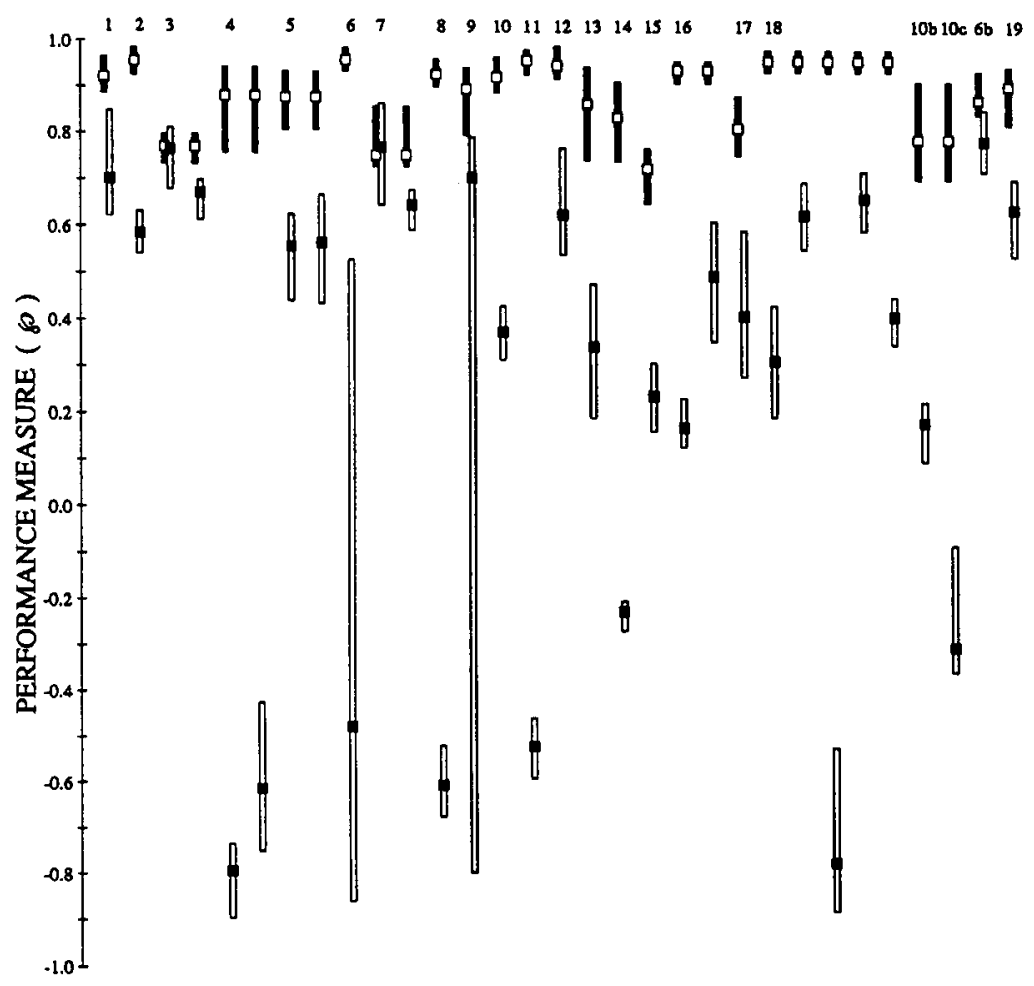

Figure 1. Results of CWA and BAM with three-point bins (A) (B) using the best fit alignment for distinguishing VT from SR (group 1). The ranges of $\rho$ during SR/AF is shown in white, with a black box at the mean, while the ranges of $\rho$ for VT is shown in black, with a white box at the mean. 


\section{PATIENT}

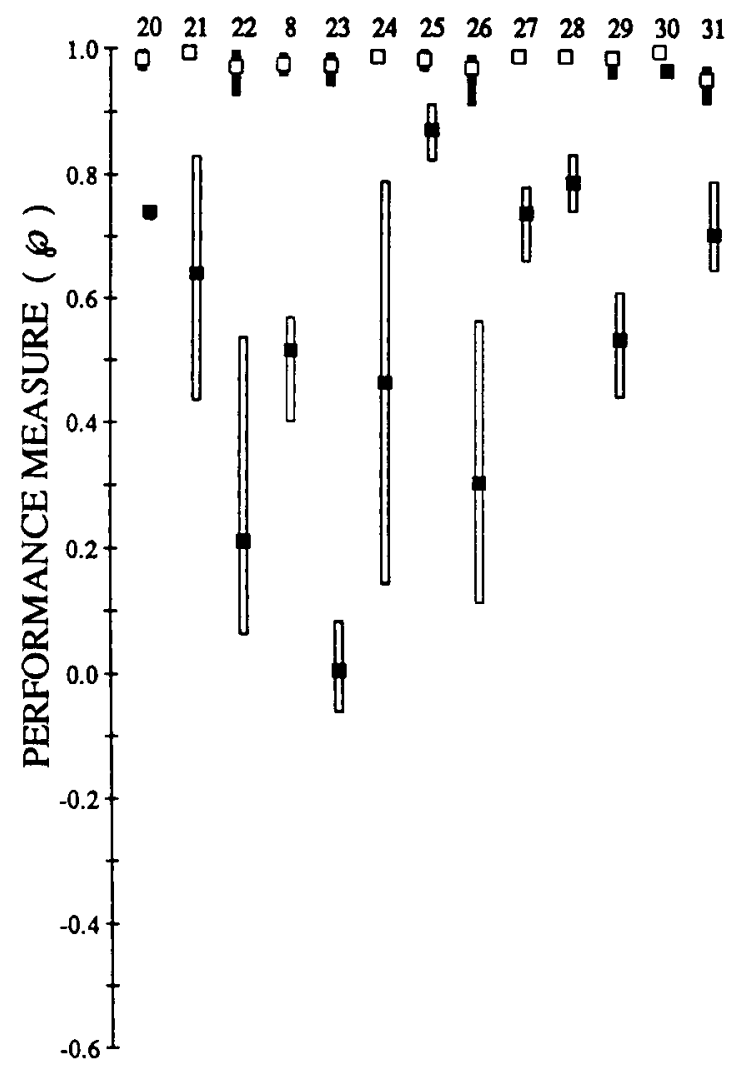

PATIENT

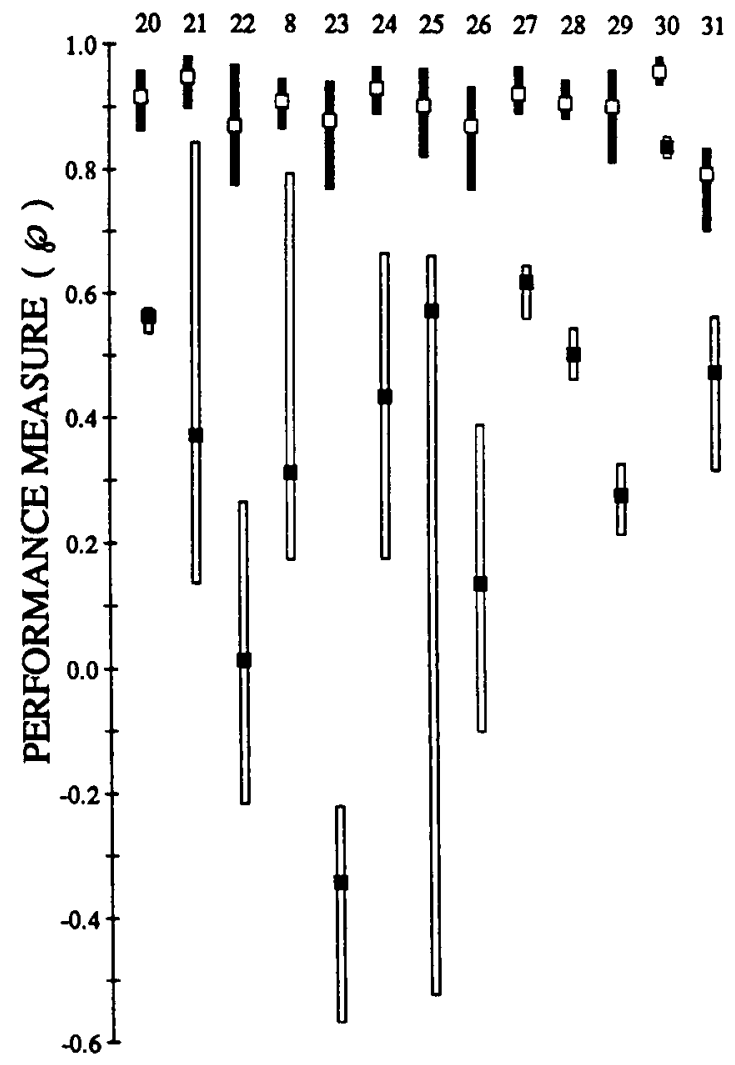

Figure 2. Results of CWA and BAM with three-point bins $(A)(B)$ using the best fit alignment for distinguishing BBB from NSR (group 2). The ranges of $\rho$ during NSR is shown in white, with a black box at the mean, while the ranges of $\rho$ for BBB is shown in black, with a white box at the mean.

\section{Discussion}

Ideally, a universal threshold would be preferable to a patient-specific threshold for separating diverse cardiac dysrhythmias. However, the critical features of intracardiac electrogram morphology that determine accurate discrimination between diverse cardiac dysrhythmias remain elusive.

The present study was limited to an analysis of bipolar intracardiac electrograms. Whether bipolar electrograms are preferrable for discriminating between dysrhythmias remains to be determined. Preliminary work from our laboratories would suggest that similar rates of success in discriminating VT from SR are achievable using timedomain analysis of either bipolar or unipolar elec- trograms in a population of patients with inducible, sustained monomorphic VT. In individual patients within that population, however, either bipolar or unipolar analysis may be preferable for maximizing the difference between SR and VT electrograms. ${ }^{11}$

The results of the present study demonstrate that time-domain techniques such as CWA are reliable for discrimination of retrograde atrial activation, paroxysmal BBB, and VT from SR. The similarity of performance of the BAM in this study supports the feasibility of developing alternative techniques which are also independent of electrogram amplitude and baseline fluctuations and have the added advantage of requiring less computational time without sacrificing diagnostic ac- 
PATIENT

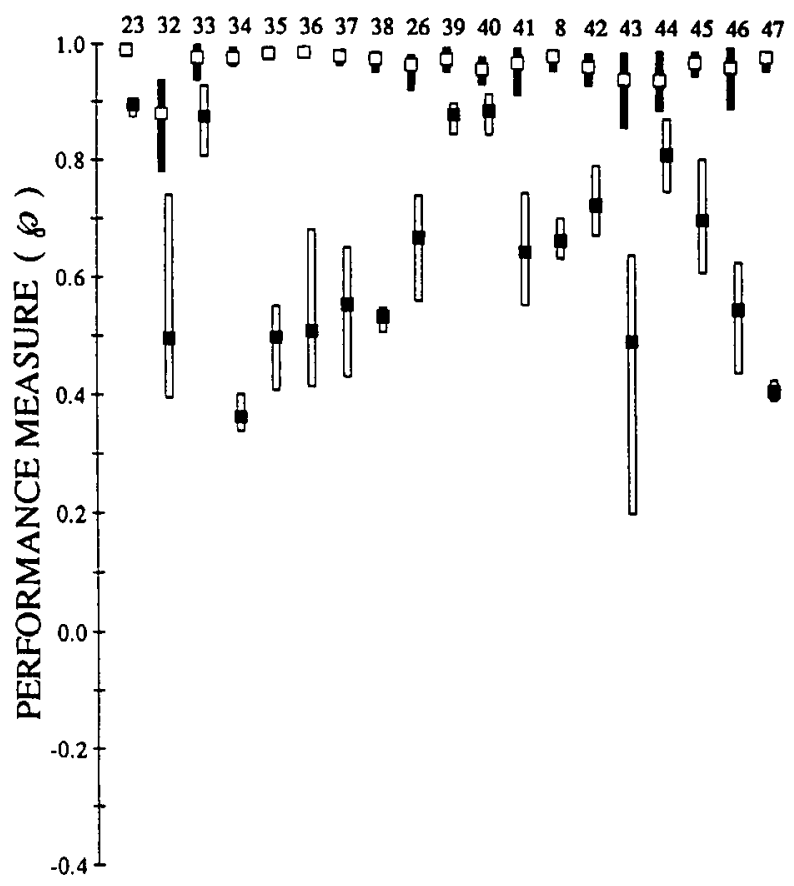

PATIENT

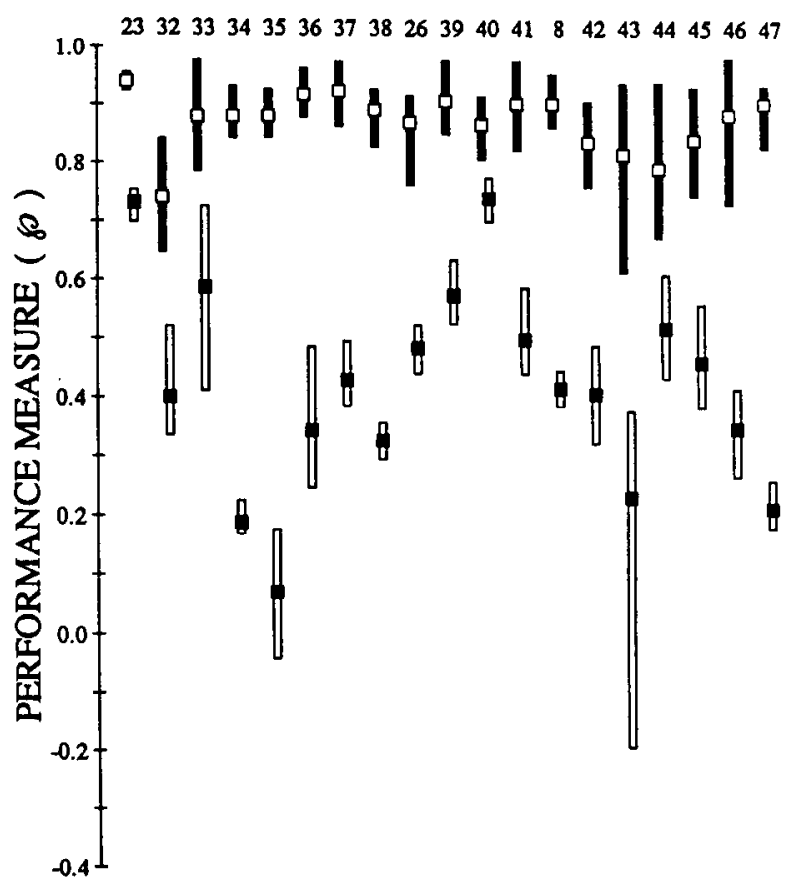

Figure 3. Results of CWA and BAM with three-point bins $(A)(B)$ using the best fit alignment for distinguishing RAA from AAA (group 3). The ranges of $\rho$ during AAA is shown in white, with a black box at the mean, while the ranges of $\rho$ for RAA is shown in black, with a white box at the mean.

curacy. ${ }^{12}$ Further reduction of time and power constraints may make real-time morphology analysis of intracardiac electrograms feasible for automatic antitachycardia and cardioverter-defibrillator devices.

\section{References}

1. Manz M, Gerckens U, Luderitz B. Erroneous discharge from an implanted automatic cardioverter/ defibrillator during supraventricular tachyarrhythmia-induced ventricular fibrillation. Am J Cardiol 1986; 7:343.

2. Platia EV, Griffith LSC, Reid PR, et al. Complications with the automatic implantable cardioverter defibrillator. (abstract) J Am Coll Cardiol 1986; $2: 200$.

3. Gabry MD, Brodman R, Johnston D, et al. Automatic implantable cardioverter-defibrillator: Patient survival, battery longevity, and shock delivery analysis. J Am Coll Cardiol 1987; 9:1349-1356.

4. Steinberg JS, Sugalski JS, Haratonic K. Cardiac rhythm precipitating automatic-implantable car-
Acknowledgments: The authors wish to thank Colleen Hoover, B.S., Judy Jaffke, R.T.(R), and Barbara Nagrant, R.C.V.T., for their technical assistance in the Cardiac Electrophysiology Laboratory, and Ms. Debbie Laird for preparation of this manuscript.

dioverter defibrillator discharge in outpatients: Observations from transtelephonic recordings. (abstract) Circulation 1989; 80:530.

5. McAlister H, Klementowicz PT, Calderon EM, et al. Atrial electrogram analysis: Antegrade vs retrograde. PACE 1988; 11:1703-1707.

6. Pannizzo F, Amikam S, Bagwell P, et al. Discrimination of antegrade and retrograde atrial depolarization by electrogram analysis. Am Heart J 1986; 112:780-786.

7. Langberg J, Gibb W, Auslander D, et al. Identification of ventricular tachycardia with use of the morphology of the endocardial electrogram. Circulation 1988; 77:1363-1369.

8. Timmis G, Westveer D, Bakalyar D, et al. Discrimination of antegrade from retrograde atrial electro- 
DICARLO, ET AL.

grams for physiologic pacing. PACE 1988; 11:130140.

9. Lin D, DiCarlo LA, Jenkins JM. Identification of ventricular tachycardia using intracavitary ventricular electrograms: Analysis of time and frequency domain patterns. PACE 1988; 11:15921606.

10. Davies DW, Wainwright RJ, Tooley MA, et al. Detection of pathological tachycardia by analysis of electrogram morphology. PACE 1986; 9:200-208.
11. Greenhut SE, Throne RD, Jenkins JM, et al. A comparative analysis of unipolar versus bipolar intraventricular electrograms for detection of ventricular tachycardia. (abstract) PACE 1990; 13:496.

12. Throne RD, Jenkins JM, Winston SA, et al. Derivative area method: A new technique for detecting ventricular tachycardia. (abstract) Circulation 1989; 80:658. 
Copyright of Pacing \& Clinical Electrophysiology is the property of Blackwell Publishing Limited and its content may not be copied or emailed to multiple sites or posted to a listserv without the copyright holder's express written permission. However, users may print, download, or email articles for individual use. 